\title{
Laminar Flow of a Sheared Vortex Crystal: Scars in Flat Geometry
}

\author{
M.-Carmen Miguel, ${ }^{1}$ Adil Mughal, ${ }^{2,4}$ and Stefano Zapperi ${ }^{3,4}$ \\ ${ }^{1}$ Departament de Física Fonamental, Facultat de Física, Universitat de Barcelona, Av. Diagonal 647, E-08028, Barcelona, Spain \\ ${ }^{2}$ Institute of Mathematics and Physics, Aberystwyth University Penglais, Aberystwyth, Ceredigion, United Kingdom \\ ${ }^{3}$ CNR-Consiglio Nazionale delle Ricerche, IENI, Via R. Cozzi 53, 20125 Milano, Italy \\ ${ }^{4}$ ISI Foundation, Viale San Severo 65, 10133 Torino, Italy \\ (Received 5 November 2010; published 15 June 2011)
}

\begin{abstract}
We consider the laminar flow of a vortex crystal in the Corbino disk geometry. Laminar flow can be induced by thermal fluctuations melting the crystal, but also by shear stress after applying a large current at zero temperature. While the velocity profile is the same in the two cases, the underlying vortex structure is completely different. A vortex crystal in this geometry can flow in a laminar fashion whenever the appropriate curvature is established in the vortex lattice. This curvature requires the presence of geometrically necessary disclinations, which here migrate from the boundary to the bulk of the crystal in the form of current-induced grain boundary scars in flat geometry. We provide an estimate of the characteristic current needed to initiate such a laminar flow regime in the vortex crystal and show that the result is in good agreement with simulations.
\end{abstract}

DOI: 10.1103/PhysRevLett.106.245501

PACS numbers: 61.72.Lk, 74.25.Uv

Under small shear stress, most liquids exhibit a laminar flow response: the local fluid velocity is proportional to the local force and they flow orderly in layers. Conversely, crystals do not flow at small stress, but deform elastically until the stress is large enough to cause plastic or irreversible flow. Notwithstanding, at very high loads crystals are sometimes observed to shear melt [1], which leads again to a laminar flow regime. To understand any microstructural differences between laminar flow in liquid and crystal phases it is necessary to observe atomic arrangements and the microscopic topological structure of the system while it is sheared. In this respect, self-assembled structures of artificial atoms [2], such as synthetic nanocrystals, magnetic colloids, charged particles in Coulomb crystals, proteins and surfactants, or vortices in type II superconductors and in Bose-Einstein condensates, represent ideal systems for this purpose. In particular, experiments and numerical simulations have suggested that shear-melting occurs in driven colloidal crystals $[1,3]$ and superconducting vortex lattices [4].

Experiments of superconducting vortex flow in the Corbino geometry display intriguing dynamic phases as a function of temperature and applied current. López et al. [5] have evaluated the vortex velocity profiles after measuring the voltage drop across a series of contacts placed radially on a superconducting disk. For low currents and temperatures, vortices move as a rigid solid. When the current exceeds a threshold value $I_{0}$, the vortex crystal cannot sustain the shear stress induced by the resulting inhomogeneous Lorentz force and the response becomes plastic. Finally, at higher currents the flow is laminar. Theoretical [6,7] and numerical [8] studies have clarified the role played by topological defects in these transitions, revealing that when the flow is laminar dislocations are arranged into long radial grain boundary (GB) scars [8]. Similar dislocation scars have received much attention in recent years in the study of crystalline materials on curved manifolds. In these systems, the interplay of crystalline order and the curvature of the substrate, leads to the appearance of stable GB scars formed by strings of dislocations attached to curvature-induced disclinations [9]. By tuning the global constraints, it is possible to dramatically influence collective properties, such as morphology $[10,11]$ and thermodynamic response [12,13]. As such these systems are at the forefront in the drive to fabricate new nanoscale functional materials [14-16]. Remarkably, the dislocation patterns observed in these equilibrium structures lying on curved geometries appears to be very similar to those we observed in nonequilibrium vortex flow, although in flat space.

In this Letter, we report the results of numerical simulations for vortex dynamics in the Corbino disk geometry in conditions giving rise to laminar flow, either by increasing temperature at low driving current or by increasing current at zero temperature. We show that while the velocity profiles are very similar in the two cases, the topological structure of the vortex array is completely different. At high temperatures, the vortex lattice melts and disclinations and dislocations are homogeneously spread throughout the disk while at low temperatures and high enough driving currents the crystal maintains a polycrystallinelike order with the formation of radial GB scars.

We consider a set of $N$ rigid vortices confined to the surface of a disk of radius $R$. The equation of motion for each vortex $i$ at a position $\mathbf{r}_{i}$ is

$$
\Gamma \frac{d \mathbf{r}_{i}}{d t}=\sum_{j} \mathbf{f}_{v v}\left(\mathbf{r}_{i}-\mathbf{r}_{j}\right)+\mathbf{f}_{L}\left(\mathbf{r}_{i}\right)+\mathbf{f}_{B}\left(\mathbf{r}_{i}\right)+\mathbf{f}_{T}\left(\mathbf{r}_{i}, t\right),
$$


where $\Gamma$ is an effective vortex friction. The pair interaction between two vortices is given by $\mathbf{f}_{v v}(\mathbf{r})=A K_{1}(|\mathbf{r}| / \lambda) \hat{\mathbf{r}}$ where $A=\phi_{0}^{2} /\left(8 \pi^{2} \lambda^{3}\right), \lambda$ is the London penetration length, $K_{1}$ is a Bessel function, and $\phi_{0}$ is the quantized magnetic flux carried by the vortices. In the Corbino geometry, the Lorentz force is given by $\mathbf{f}_{L}(r)=\hat{\boldsymbol{\theta}} \phi_{0} J(r) / c$ where $c$ is the speed of light, $\hat{\boldsymbol{\theta}}$ is the azimuthal unit vector. and the radial current density is $\mathbf{J}(r)=\hat{\mathbf{r}} I /(2 \pi r t)$, where $t$ is the thickness of the superconducting disk. We choose as units of space and time $\lambda$ and $t_{0}=\Gamma \lambda / A$ respectively, and we measure the current $I$ in units of $\phi_{0} /(2 \pi c t \lambda A)$. The $N$ vortices are confined inside the disk by a radial confining force $\mathbf{f}_{B}=-g \exp \left[-(R-r) / r_{0}\right] / r_{0} \hat{\mathbf{r}}$ with $r_{0}=0.1 \lambda$ and $g / A=1$. A similar force is also imposed at the inner wall close to the disk center (at $r=r_{0}$ ) thus avoiding the singularity of the Lorentz force at $r=0$. Finally, $\mathbf{f}_{T}\left(\mathbf{r}_{\mathrm{i}}, t\right)$ is a Gaussian uncorrelated random force that takes into account the effect of temperature. In order to comply with the fluctuation-dissipation theorem, we choose the correlation as $\left\langle\mathbf{f}_{T}\left(\mathbf{r}_{i}, t\right) \mathbf{f}_{T}\left(\mathbf{r}_{j}, t^{\prime}\right)\right\rangle=2 \Gamma k_{B} T \delta_{i j} \delta\left(t-t^{\prime}\right)$, where $T$ is the temperature and $k_{B}$ is the Boltzmann constant.

The coupled equations Eq. (1) for $i=1, \ldots, N$ are integrated numerically at $T=0$ with an adaptive step size fifth-order Runge-Kutta method with precision $10^{-6}$. At $T>0$, we use a simpler Euler integration scheme. At $T=0$, we study the response of the system as a function of $I$ for systems of radius $R=18 \lambda$ and $R=36 \lambda$ and various values of $N$. In each case a value for $I$ is chosen and the system is allowed to evolve for 10000 time steps, for roughly the first 1000 time steps the system displays transient behavior before settling down into a steady state.

As already shown in Ref. [8], the vortex lattice rotates rigidly below a critical current $I_{0}$ after which it deforms plastically. Here, we focus on the behavior of a second threshold current $I_{1}$, above which vortex flow becomes laminar; i.e., the velocity profiles $v(r)$ follow the driving force profile decaying as $1 / r$. Above $I_{1}$ we observe vortex flow in the azimuthal direction in the form of uncorrelated rings. This type of flow allows an efficient release of the shear stress concentration inside the crystal. This can be seen in Fig. 1(a). In addition, we simulate the model at a lower current $I<I_{1}$, so that at $T=0$ the flow would be plastic. By increasing the temperature, we observe a crossover to a laminar flow profile [Fig. 1(b)], but with a completely different topological microstructure.

To identify the most relevant microstructural features of laminar flow induced by increasing current or temperature, we analyze the topological properties of the vortex array in the corresponding phases. In Figs. 1(c) and 1(d), we show the vortex positions at single snapshots within the laminar phase for a system consisting of $N=2032$ vortices in a disk of radius $R=18 \lambda$. The number of nearest neighbors for each vortex has been identified using a Delaunay triangulation package. Notice the striking difference between the topology in Fig. 1(d), obtained at high
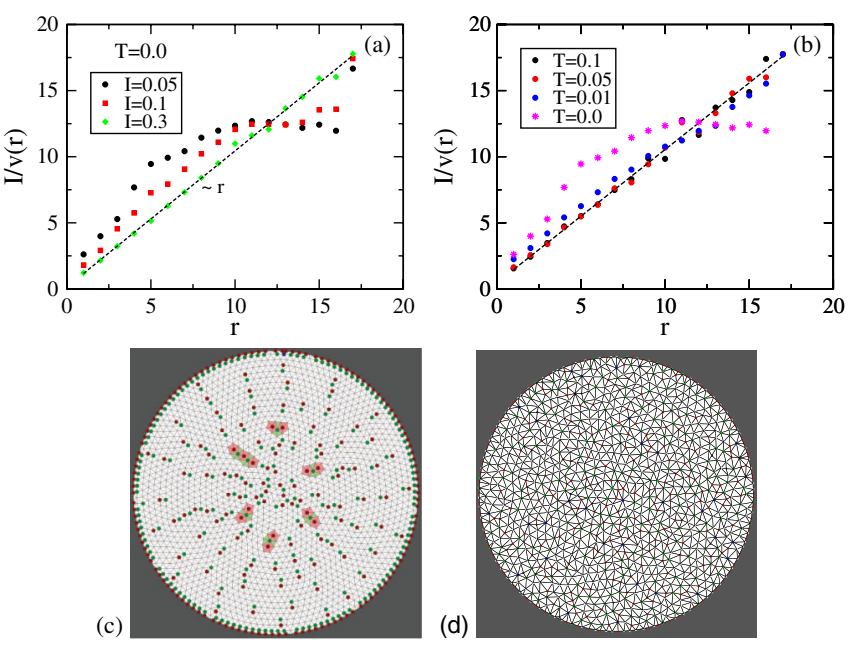

FIG. 1 (color online). (a) The evolution of the inverse velocity profiles as a function of the applied current at $T=0$. As the current increases the inverse profile becomes linear as expected for laminar flow. (b) The evolution of the inverse velocity profiles as a function of temperature. At high temperatures, we also observe a laminar flow profile. (c) Delaunay triangulation of $N=2032$ vortices at $T=0$ and $I=0.275$. Notice the dislocations arranged into GB scars and six excess disclinations forming part of that structure in the interior of the disk. (d) Delaunay triangulation of the vortices at $T=0.1$ and $I=0.05$. Topological defects appear all over indicating a liquidlike structure.

temperature and low current, and the polycrystalline phase at $T=0$ in Fig. 1(c). In the latter, it can be seen that while most vortices have six nearest neighbors there are also some which have an anomalous coordination number. Such points are topological defects of the triangular lattice known as $60^{\circ}$ wedge disclinations and have a positivenegative topological charge. A geometrical constraint, due to Euler's theorem [17], states that the total number of pentagonal regions must always exceed the number of heptagonal regions by six in two dimensions. In most cases disclinations are tightly bound into oppositely charged pairs forming dislocations, another type of topological defect whose number is, however, not constrained by topology. In Fig. 1(d), these topological defects are spread all over the system in a disordered fashion.

The absence of long-range order at high temperatures is a signature of the liquid phase, where laminar flow is expected. In the crystalline phase at $T=0$, however, the observation of laminar flow is more intriguing. In the absence of currents, the interior of the vortex lattice is free of defects and the six geometrically necessary disclinations are located right at the edge of the disk. This arrangement minimizes the enormous elastic strain generated by the disclinations, while their presence at the edge imposes a curvature on the lattice making it commensurate with the circular boundary. The elastic stress is further reduced by attaching these irreducible disclinations to a screening cloud of dislocations. Together these form GB 
scars oriented along the circumference of the disk, each one emanating from one of the extra six positive disclinations. In the high current phase, however, dislocations are found in large numbers throughout the system forming radial GBs. Moreover most dislocations now have the Burgers vector in the azimuthal direction. Dislocation gliding in the tangential direction, i.e., in the direction of the driving force, makes possible a laminar velocity profile and the most efficient release of shear stress in the crystal. But dislocation glide along the tangential direction is only possible because the bending of one of the crystallographic directions in the triangular lattice into a circumference is now maintained steadily throughout the disk [see Fig. 1(c)]. Furthermore, the curvature of these lattice lines demands that the six topologically necessary disclinations are located deep in the interior of the disk. These have been highlighted in Fig. 1(c). An excess positive disclination charge in the interior of a disk geometry has the effect of bending lattice lines [18], which permits dislocations to glide in circular circuits around the center of the system. Thus the vortex lattice can be divided into a series of concentric rings which slip past each other giving rise to the observed laminar flow.

These processes must occur simultaneously right above $I_{1}$. Thus, based upon the previous observations, we can propose an argument to determine the second current threshold $I_{1}$ for steady gliding along the tangential direction. We propose to identify $I_{1}$ as the current at which new dislocations pairs can be nucleated throughout the entire system, up to the disk edge, by the action of the currentinduced shear stress. Indeed we see that GB scars only form when enough dislocations with a Burgers vector in the azimuthal direction are present in the system up to the disk edge. Unlike dislocation pairs nucleated very close to the disk center, pairs nucleated elsewhere can unbind in any crystallographic direction allowing the beginning of the scars' inward migration. The distribution of elastic shear stress in a rotating perfect crystal in this particular geometry is given by [19]

$$
\sigma_{r \theta}(r, I)=-\frac{I B_{0}}{4 c \pi t}\left(1-\frac{r^{2}}{R^{2}+r_{0}^{2}}-\frac{R^{2} r_{0}^{2}}{R^{2}+r_{0}^{2}} \frac{1}{r^{2}}\right)
$$

where $B_{0}=n \phi_{0}$ is the average magnetic induction, $n$ the areal density of flux lines, and $r_{0}$ is the small cutoff radius used in simulations. Note that $\sigma_{r \theta}(R, I)=\sigma_{r \theta}\left(r_{0}, I\right)=0$. At $I_{0}$, we saw that the maximum shear stress close to the disk center, $\sigma_{r \theta}^{\max }\left(I_{0}\right)=-I_{0} B_{0} / 4 c \pi t$, was sufficient to nucleate new dislocation pairs there [8]. For dislocations to be nucleated throughout the system at $I=I_{1}$, up to a distance $\delta$ from the disk edge, we then require that

$$
\sigma_{r \theta}\left(R-\delta, I_{1}\right)=\sigma_{r \theta}^{\max }\left(I_{0}\right) .
$$

Expanding (2) to first order in $\delta$ and solving for $I_{1}$ yields,

$$
I_{1}=I_{0} \frac{R}{2 \delta} \text {. }
$$

From our numerical simulations we can now identify the value of the current $I_{1}$ at which the second jump in the resistance occurs. These values for Corbino disks with radius $R=18 \lambda$ and $R=36 \lambda$, over a range of densities, are plotted in Fig. 2. Also shown is the theoretical line generated using Eq. (3) with the best fit obtained when $\delta=1.81 a$. The theoretical estimate of $I_{0}=C c_{66}(a / \lambda) / \tilde{c}_{66}$ was found in [8], where $a$ is the lattice spacing, $c_{66}(a / \lambda)=$ $\tilde{c}_{66} f(a / \lambda)$ is the shear modulus of the vortex lattice, $\tilde{c}_{66}=B_{0} \phi_{0} /(8 \pi \lambda)^{2}$ is the shear modulus in the long wavelength limit, and $f(a / \lambda)$ is a function describing the dependence of the shear modulus on $a$ [20]. Moreover, $C=\sqrt{2} b / 4 \pi r_{d}$ was a theoretically derived constant (where we set the Burgers vector modulus $b=a$ and the dislocation dipole extent $r_{d} \approx 2-3 a$ ).

The emergence of the second transition at $I_{1}$ is, as discussed, associated with the migration of excess disclination structures towards the interior of the disk. This process is slow and difficult to describe analytically, because disclinations do not move alone but are assisted by the progressive formation of extended grain boundaries emanating from them.

In order to confirm these ideas, we compute the center of mass $(\mathrm{COM})$ of excess positive disclination charge structures as a function of current (see Fig. 3). Since the slip velocity decreases with the distance to the center, the structure of grain boundary scars is not rigid but constantly rearranging in time. Thus the COM is a highly fluctuating quantity. In Fig. 3, we plot (i) the minimum radius within which the 6 COMs are contained-out of 1000 snapshots for a given value of the current, and (ii) the average radius within which the $6 \mathrm{COMs}$ are contained for that value of the current. For the case of $R=18 \lambda$ and $N=2032$ shown in Fig. 1(c), we see that before $I_{0}$ all the positive disclinations are pinned firmly on the outer edge of the cluster, after which there is a small drop in the smallest radius at which the six disclinations can be found. This is because dislocations nucleated in the interior, upon reaching the

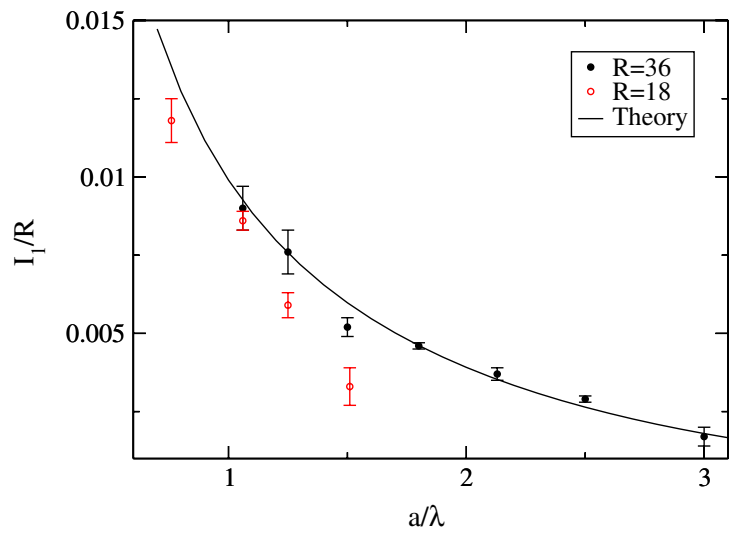

FIG. 2 (color online). A fit of Eq. (3) with $\delta=1.81 a$ against the values of the transition current $I_{1}$, as obtained by numerical experiments for systems of radius $R=18 \lambda$ and $R=36 \lambda$. 


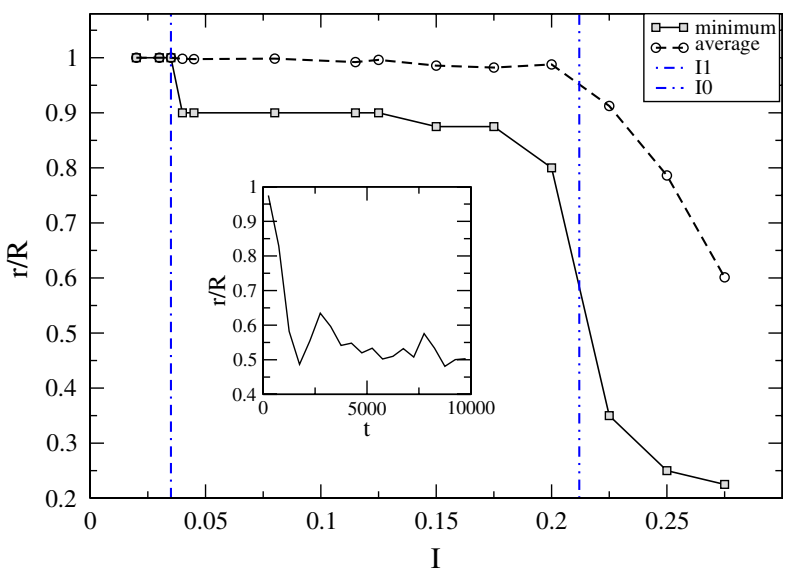

FIG. 3 (color online). Average radial position of the COM of the excess disclination structures as a function of the applied current. We plot both the average and the smallest radial position reached during the simulation. Inset: Average radial position as a function of time at $I=0.275$, showing how disclination structures slowly migrate towards the interior of the Corbino disk. The data points have been averaged over a time window of length $\Delta t=50$.

disk edge due to plastic slip along the radial direction, sometimes become attached to the disclination structures present there. Near $I_{1}$ there is a dramatic drop in both the average and minimum radii, and as the current is increased beyond $I_{1}$ it is found that, on average, the COMs of the six disclination structures are found further and further towards the interior of the disk. In the insert of Fig. 3 we show that the position of the disclination's COM migrates towards the center of the disk. These results hold qualitatively true for low but finite $T$, but we expect to find lower $I_{1}$ values for thermally softened vortex lattices as well as higher defect densities and slower defect dynamics.

In conclusion, we have shown that laminar flow occurs both in sheared liquid and crystalline vortex matter phases in the Corbino disk geometry. While in liquids, the shear profile is allowed by the loss of long-range order, in crystals it is due to the glide motion of dislocations forming radial GB scars when the required curvature of the crystal lines is provided by the migration of six excess disclination structures towards the interior of the disk. Our results provide an intriguing analogy between the equilibrium topological structure of a crystal on a three-dimensional curved geometry and the nonequilibrium structure of a sheared crystal in flat geometry. As in other out of equilibrium phase transformations, like in shear melting or shear banding, here the topological transition to the GB scar phase, which should be energetically forbidden in equilibrium conditions, is controlled by a current-induced shear stress, and it should be relevant for other crystalline thin films of current interest.

We thank P. Moretti for useful discussions. M.C.M. acknowledges financial support by the Ministerio de Ciencia e Innovación (Spain), Grants No. FIS2007-66485-C02-02, FIS2010-21781-C02-02 and No. HH2008-0002, and by the Generalitat de Catalunya and the Ministerio de Educación y Ciencia (Programa I3) for additional funding. M. C.M. also wishes to thank the hospitality of the ISI Foundation (Turin, Italy), where part of this work was carried out.

[1] B. J. Ackerson and N. A. Clark, Phys. Rev. Lett. 46, 123 (1981).

[2] C. Murray, C. Kagan, and M. Bawendi, Annu. Rev. Mater. Sci. 30, 545 (2000).

[3] M. J. Stevens, M. O. Robbins, and J. F. Belak, Phys. Rev. Lett. 66, 3004 (1991).

[4] A. E. Koshelev and V. M. Vinokur, Phys. Rev. Lett. 73, 3580 (1994).

[5] D. López, W. K. Kwok, H. Safar, R. J. Olsson, A. M. Petrean, L. Paulius, and G. W. Crabtree, Phys. Rev. Lett. 82, 1277 (1999).

[6] M. C. Marchetti and D. R. Nelson, Physica C (Amsterdam) 330, 105 (2000).

[7] P. Benetatos and M. C. Marchetti, Phys. Rev. B 65, 134517 (2002).

[8] M. C. Miguel and S. Zapperi, Nature Mater. 2, 477 (2003).

[9] M. J. Bowick and L. Giomi, Adv. Phys. 58, 449 (2009).

[10] V. Vitelli, J. B. Lucks, and D. R. Nelson, Proc. Natl. Acad. Sci. U.S.A. 103, 12323 (2006).

[11] A. M. Turner, V. Vitelli, and D. R. Nelson, Rev. Mod. Phys. 82, 1301 (2010).

[12] C. D. Modes and R.D. Kamien, Phys. Rev. Lett. 99, 235701 (2007).

[13] C. D. Modes and R. D. Kamien, Phys. Rev. E 77, 041125 (2008).

[14] D. R. Nelson, Nano Lett. 2, 1125 (2002).

[15] A. R. Bausch, M. J. Bowick, A. Cacciuto, A. D. Dinsmore, M. F. Hsu, D. R. Nelson, M. G. N. A. Travesset, and D. A. Weitz, Science 299, 1716 (2003).

[16] R. D. Kamien, Science 299, 1671 (2003).

[17] A. A. Koulakov and B.I. Shklovskii, Phys. Rev. B 57, 2352 (1998).

[18] A. Mughal and M. A. Moore, Phys. Rev. E 76, 011606 (2007).

[19] A. Furukawa and Y. Nisikawa, Phys. Rev. B 73, 064511 (2006).

[20] M.C. Miguel and M. Kardar, Phys. Rev. B 62, 5942 (2000). 